\title{
Multi-Dimensional LUT-based Digital Predistorter for Concurrent Dual-Band Envelope Tracking Power Amplifier Linearization
}

\author{
Quynh Anh Pham*, David López-Bueno **, Teng Wang*, Gabriel Montoro* and Pere L. Gilabert* \\ *Dept. Signal Theory and Comm., Universitat Politècnica de Catalunya (UPC), Castelldefels, Spain \\ \#Centre Tecnològic de Telecomunicacions de Catalunya (CTTC), Castelldefels, Spain
}

\begin{abstract}
This paper presents a multi lookup table (LUT) implementation scheme for the 3D distributed memory polynomial (3D-DMP) behavioral model used in Digital Predistortion (DPD) linearization for concurrent dual-band envelope tracking (ET) power amplifiers (PAs). The proposed 3DDistributed Memory LUTs (3D-DML) architecture is suitable for efficient FPGA implementation. In order to optimize the linearization performance as well as to reduce the number of resources of the 3D-DML model, a new variant of the Orthogonal Matching Pursuit (OMP) algorithm is proposed to properly select the best LUTs. Experimental results show that the proposed strategy reduces the number of LUTs (i.e. the number of coefficients) while meeting the targeted linearity levels.
\end{abstract}

Index Terms - Envelope tracking, digital predistortion, lookup tables, power amplifier.

\section{INTRODUCTION}

In concurrent dual-band (DB) transmissions with envelope tracking (ET) power amplifiers (PAs), several linearization challenges have to be addressed. For example, assuming that the nonlinear distortions of concern are those that arise close to the band of interest, it is possible to design specific DPD linearizers for each band, taking into account possible cross-band intermodulation distortion between bands. Moreover, since the envelope modulators efficiency is kept along a limited bandwidth, we cannot supply the power required by the transistor at the same speed of the DB signal's envelope. Consequently, as explained in [1], we can use a slower version of the instantaneous DB envelope to supply the PA (e.g., sum of the modulus of both baseband signals). Therefore, in concurrent DB ET PAs, specific DPD linearizers are designed for each band to compensate for the in-band and cross-band intermodulation distortion as well as for the slow-envelope dependent distortion that appears when supplying the PA with a slower version of the DB envelope.

In this paper we propose a multi-LUT architecture targeting a FPGA implementation of the 3-D distributed memory polynomial (3D-DMP) DPD proposed by the authors in [1]. The proposed 3-D distributed memory LUT (3D-DML) architecture follows the linear/bilinear interpolation and extrapolation presented by Molina et al. in [2]. In addition, a modified version of the Orthogonal
Matching Pursuit (OMP) algorithm [3] is proposed to properly select the most relevant LUTs of the 3D-DML model. Experimental results to validate the 3D-DML DPD were obtained using the remoteUPCLab test bed, built by the authors to organize the IMS2017 DPD student design competition [4].

The remainder of this paper is organized as follows. Section II presents the 3D-DML model. Section III describes the proposed best LUTs selection method in details. Section IV demonstrates the measurement results of the proposed selection method on 3D-DML model. Section V gives the conclusions.

\section{3D-DML Digital Predistorter}

To derive the 3D-DMP DPD in [1] into a set of LUTs for FPGA implementation, we have considered the LUT linear/bilinear interpolation and extrapolation presented in [2]. In a concurrent DB transmission, each band will be predistorted by its particular DPD. The input-output relationship in the 3D-DML DPD for Band 1 is defined as

$$
\begin{aligned}
& x_{1}[n]=\sum_{i=0}^{N_{1}-1} u_{1}\left[n-\tau_{i}^{u_{1}}\right] f_{\bar{\Phi}_{1, i}}\left(\left|u_{1}\left[n-\tau_{i}^{u_{1}}\right]\right|\right)+ \\
& \sum_{i=1}^{N_{2}-1} \sum_{j=1}^{M_{2}-1} u_{1}[n] f_{\bar{\Phi}_{1, i, j}}\left(\left|u_{1}\left[n-\tau_{i}^{u_{1}}\right]\right|,\left|u_{2}\left[n-\tau_{j}^{u_{2}}\right]\right|\right)+ \\
& \sum_{i=1}^{N_{3}-1} \sum_{k=1}^{K_{3}-1} u_{1}[n] f_{\bar{\Phi}_{1, i, k}}\left(\left|u_{1}\left[n-\tau_{i}^{u_{1}}\right]\right|, E\left[n-\tau_{k}^{e}\right]\right)
\end{aligned}
$$

where $N_{1}, N_{2}$ and $N_{3}$ are the numbers of delays of the input signal at each branch; $M_{2}$ is the number of delays of the interference signal $u_{2}[n] ; K_{3}$ is the number of delays of the supply envelope $E[n] ; \tau^{u_{1}}, \tau^{u_{2}}$ and $\tau^{e}$ (with $\tau^{u_{1}, u_{2}, e} \in \mathbf{Z}$ and $\tau_{0}^{u_{1}, u_{2}, e}=0$ ) are the most significant sparse delays of the input $\left(u_{1}[n]\right)$, interference signal $\left(u_{2}[n]\right)$ and envelope $(E[n]) ; f_{\bar{\Phi}}(u)$ and $f_{\bar{\Phi}}(u, v)$ are 1-D LUT and 2-D LUT respectively, presented in [2] and further described later in this section. The DPD for Band 2 can be similarly modeled as in (2) with $u_{2}[n]$ and $u_{1}[n]$ are the input and interfering signals.

On the one hand, the 1-D LUT in (1) is a piecewise linear complex function, defined in (2) as the linear com- 
bination of $N$ basis functions.

$$
f_{\bar{\Phi}}(u)=\sum_{i=0}^{N-1} \varphi_{i} \Lambda_{g(i, N)}(u-i \delta)
$$

where $u$ is a real number, $g(i, N)=\left\{\begin{array}{l}0, i<N-2 \\ 1, i=N-2 \\ 2, i=N-1\end{array}\right.$; $\delta=\max (u) /(N-1)$ is the width of each region on the real interval at which function $f_{\bar{\Phi}}(u)$ is defined; $\Lambda_{0}(u)$ defined in (3) is the interpolation basis function on the interval $[0,(N-1) \delta]$; while $\Lambda_{1}(u)$ in (4) and $\Lambda_{2}(u)$ in (5) are extrapolation basis functions on the interval $[(N-$ 1) $\delta, \infty]$. Finally, $\varphi_{i}$ are the coefficients of the picewise complex function.

$$
\begin{aligned}
& \Lambda_{0}(u)=\left(1-\frac{|u|}{\delta}\right) w\left(\frac{|u|}{\delta}\right) ; w(u)= \begin{cases}1, & 0 \leq u \leq 1 \\
0, & \text { otherwise }\end{cases} \\
& \Lambda_{1}(u)=\left(1-\frac{|u|}{\delta}\right) s(u+\delta) ; s(u)= \begin{cases}1, & u \geq 0 \\
0, & \text { otherwise }\end{cases} \\
& \Lambda_{2}(u)=\left(1+\frac{u}{\delta}\right) s(u+\delta) ; s(u)= \begin{cases}1, & u \geq 0 \\
0, & \text { otherwise }\end{cases}
\end{aligned}
$$

On the other hand, the 2-D LUT in (1) is defined by a piecewise bilinear complex function as follows,

$$
\begin{array}{r}
f_{\bar{\Phi}}\left(u_{1}, u_{2}\right)=\sum_{i=0}^{N_{1}-1} \sum_{j=0}^{N_{2}-1} \varphi_{i, j} \Gamma_{g\left(i, N_{1}\right), g\left(j, N_{2}\right)} \\
\left(u_{1}-i \delta_{1}, u_{2}-j \delta_{2}\right)
\end{array}
$$

where $\Gamma_{i, j}\left(u_{1}, u_{2}\right)=\Lambda_{i}\left(u_{1}\right) \Lambda_{j}\left(u_{2}\right)$. Functions $g(i, N)$, $\Lambda_{0}(u), \Lambda_{1}(u), \Lambda_{2}(u), w(u)$ and $s(u)$ are defined in (2)(5). In (6), $u_{1}$ and $u_{2}$ are real numbers; $N_{1}$ and $N_{2}$ are the numbers of basis functions in the $u_{1}$ and $u_{2}$ directions; $\delta_{1}$ and $\delta_{2}$ are the widths of each region of $u_{1}$ and $u_{2}$ respectively, $\delta_{1}=\max \left(u_{1}\right) /\left(N_{1}-1\right)$ and $\delta_{2}=\max \left(u_{2}\right) /\left(N_{2}-1\right)$. Further details on the bilinear interpolation and extrapolation can be found in [2].

\section{Best LUTs Selection Method (OMP-LUT)}

When considering concurrent DB transmissions, the number of coefficients required in the DPD model to compensate for the in-band, cross-band intermodulation distortion and the slow-envelope dependent distortion in ET PAs can be significantly high. This negatively impacts on the least squares (LS) DPD model extraction because it increases the computational complexity and can drive to over-fitting and uncertainty. The sparsity of the LUTbased DPD models can be exploited to reduce the number of required basis functions or active components. For example, by using a greedy method such as Orthogonal Matching Pursuit (OMP), it is possible to obtain a sorted set of the most relevant basis functions (i.e. the OMP list) [3]. Because 3D-DML builds models in LUTs, direct application of OMP to the 3D-DML data matrix may be impractical. Thus, to retain the effect of LUTs on the 3D-DML model, we propose a method to allow doing the selection in LUTs, instead of the individual basis functions (corresponding to the columns of the 3D-DML data matrix). The proposed method is described in the following.

In order to value the significance of a LUT $i$ on the 3D-DML model, we consider the times a LUT $i$ appears in the OMP list, $t_{i}=\sum_{j=1}^{n} a_{j}$, in which $n$ is the number of basis functions (columns) of the LUT $i$; if column $j$ of LUT $i$ is in the OMP list $a_{j}=1$, otherwise $a_{j}=0$. It is also needed to $(a)$ discriminate between LUTs which have same size $n$ and appearance times $t$ in the OMP list; and $(b)$ determine a criterion to make different-size LUTs comparable. The OMP algorithm sorts the basis functions by relevance (i.e., the later a basis function appears in the list, the less significant it is). Therefore, to enable $(a)$, we give a weight $w$ to each element in the OMP list. $w$ is an assigned small positive value. For the first element in the list, its weight is the smallest one. In our experiment, the initial value $w$ is assigned to 0.01 ; and then this value is increased every position by 0.01 . The sum of weights $(s)$ of a LUT $i$ is $s_{i}=\sum_{j=1}^{n} w_{j}$, in which $w_{j}$ is the weight of the column $j$ in the LUT $i$. Given the LUT $i_{1}$ and LUT $i_{2}$ with $t_{i_{1}}=t_{i_{2}}$, if $s_{i_{1}}<s_{i_{2}}$ then the LUT $i_{1}$ is considered to be more relevant than the LUT $i_{2}$, and vice versa. To solve $(b)$, we compute the final weight $(l)$ of a LUT $i$ as follow $l_{i}=\left(t_{i}-s_{i}\right) / n$. The greater $l$ is, the better the LUT is. The list of LUTs is ranked in decreasing order according to the value $l$ of each LUT. A small number of LUTs used as starting point is increased until meeting linearity requirements.

\section{ExPERImEntal Setup And Results}

The remoteUPCLab test bed in Fig. 1, assembled by the authors in the framework of the IMS2017 DPD student design competition [4], was used to obtain the experimental results. It consisted of a PC running MATLAB and an FTP server to allow worldwide users to connect to the equipment: (i) a Rohde \& Schwarz (R\&S) SMW200A vector signal generator, (ii) a R\&S FSW8 signal and spectrum analyzer, and (iii) the DUT consisting in a Texas Instruments LM3290-91-1EVM ET board that includes a Skyworks SKY776621 4G handset PA operated at 1950 $\mathrm{MHz}$ with dual-band LTE $10 \mathrm{MHz}$ and LTE $5 \mathrm{MHz}$ signals (with $80 \mathrm{MHz}$ spacing). In order to assess the efficiency of the OMP-LUT method for 3D-DML DPD, three different selection methods were compared: (a) LUTs selection by 


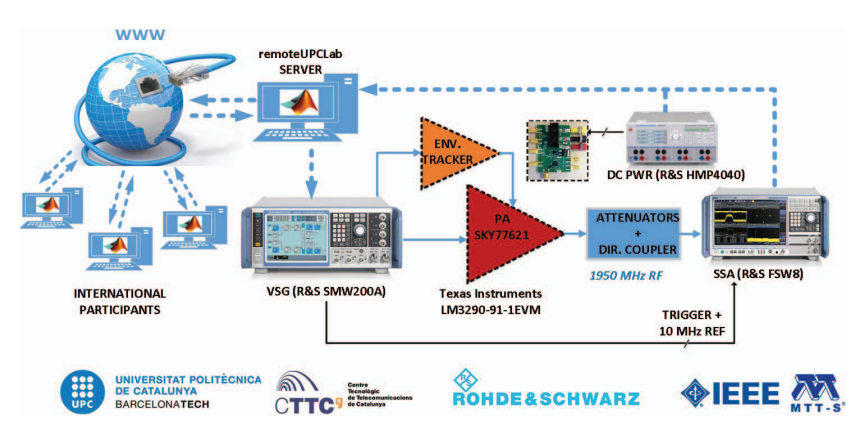

Fig. 1. Block diagram of the remoteUPCLab [4].

TABLE I

COMPARISON OF DIFFERENT OMP COEFFICIENT SELECTION METHODS FOR 3D-DML DPD.

\begin{tabular}{|c|ccccc|}
\hline Method & $\begin{array}{c}\text { Pout } \\
(\mathrm{dBm})\end{array}$ & $\begin{array}{c}\eta \\
(\%)\end{array}$ & $\begin{array}{c}\text { NMSE } \\
(\mathrm{dB})\end{array}$ & $\begin{array}{c}\text { ACPR } \\
(\mathrm{dB})\end{array}$ & $\begin{array}{c}\text { Num. } \\
\text { coeff. }\end{array}$ \\
\hline $\begin{array}{c}(a) \\
\text { No OMP }\end{array}$ & 23,1 & 18,97 & B2: $-37,67$ & B2: $-46,02$ & B2: 153 \\
\hline $\begin{array}{c}(b) \\
\text { OMP-col }\end{array}$ & 22,8 & 18,21 & B2: $-37,48$ & B2: $-45,33$ & B2: 92 \\
\hline $\begin{array}{c}(c) \\
\text { OMP-LUT }\end{array}$ & 23,0 & 18,67 & B2: $-37,14$ & B2: $-45,25$ & B2: 73 \\
\hline
\end{tabular}

sequentially adding delays, (b) OMP-col, best basis functions (or columns) selection by using OMP independently to which LUT they belong to, and (c) best LUT selection with OMP-LUT. The linearity and the power efficiency of the three methods are evaluated in Table I. The results are taken when the system meets the ACPR threshold of $-45 \mathrm{~dB}$. Table I shows that to reach $-45 \mathrm{~dB}$ of ACPR and achieve equivalent NMSE, Pout and power efficiency values, the OMP-LUT selection method uses a smaller number of LUTs, and thus coefficients, than the other two methods.

The advantage of the OMP-LUT method is also illustrated in Fig. 2. Applying the method (a), which mainly consist in adding delays sequentially, may lead to an illconditioned estimation and may require a large number of coefficients to achieve the threshold of $-45 \mathrm{~dB}$ of ACPR. Note the DPD for Band 2 for example, requiring up to 153 coefficients or, what is the same, 2 1-D LUTs and 10 2-D LUTs. When selecting the most relevant coefficients with OMP, the original universe of possible coefficients is limited to 110 coefficients for Band 1 and 119 for Band 2 . As expected, thanks to the OMP algorithm, both methods (b) and (c), allow reaching the targeted ACPR value in both bands with less coefficients than method $(a)$. However, while the OMP-col method requires 92 coefficients for both bands to get $-45 \mathrm{~dB}$ of ACPR, the proposed OMPLUT method $(c)$, allows meeting the requirements with only 62 coefficients (i.e., a 3D-DML DPD with 21 -D LUTs and 5 2-D LUTs) for Band 1 and 73 coefficients

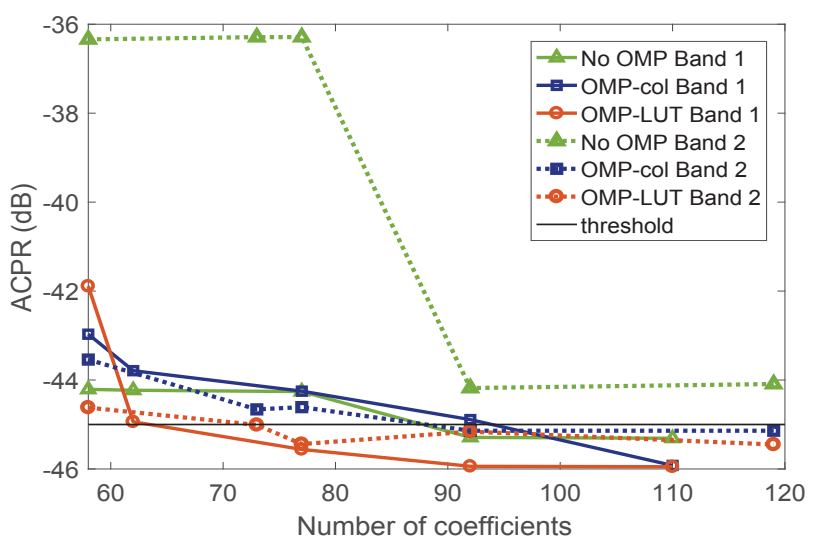

Fig. 2. ACPR versus number of coefficients for different coefficient selection methods.
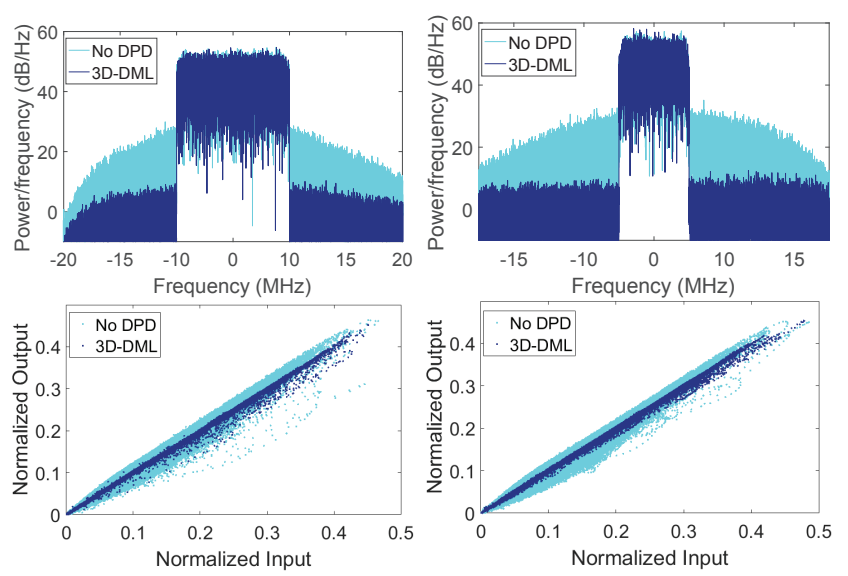

Fig. 3. Unlinearized and linearized spectra and AM-AM of Band 1 (LTE-10 MHz @ 1910 MHz) \& Band 2 (LTE-5 MHz @ 1990 $\mathrm{MHz}$ ) signals.

(i.e., a 3D-DML DPD with 3 1-D LUTs and 4 2-D LUTs) for Band 2. Fig. 3 shows the spectra and AM-AM characteristics before and after 3D-DML DPD for both bands.

\section{CONCLUSION}

We have proposed a multi-LUT approach (i.e., the 3DDML DPD) to implement a DPD capable to cope with the nonlinear distortion that arises in an ET PA under a concurrent DB transmission. In addition, we have proposed a new strategy (OMP-LUT) to select the best LUTs and minimize the required resources for FPGA implementation while meeting the required linearization specifications.

\section{ACKNOWLEDGMENT}

This work was supported by the Spanish Government (MINECO) and FEDER under projects TEC2014-58341C4-03/04-R and TEC2017-83343-C4-2-R. 


\section{REFERENCES}

[1] P. L. Gilabert and G. Montoro, "3-D Distributed Memory Polynomial Behavioral Model for Concurrent Dual-Band ET PA Linearization," IEEE Trans. on Microw. Theory and Tech., vol. 63, pp. 638-648, Feb 2015.

[2] A. Molina, K. Rajamani, and K. Azadet, "Concurrent dual-band digital predistortion using 2-d lookup tables with bilinear interpolation and extrapolation: Direct least squares coefficient adaptation," IEEE Transactions on Microwave Theory and Techniques, vol. 65, no. 4, pp. 1381-1393, April 2017.

[3] J. Reina-Tosina, M. Allegue-Martnez, C. Crespo-Cadenas, C. Yu, and S. Cruces, "Behavioral modeling and predistortion of power amplifiers under sparsity hypothesis," IEEE Transactions on Microwave Theory and Techniques, vol. 63, no. 2, pp. 745-753, Feb 2015.

[4] http://dpdcompetition.com/sdc. 